\title{
Dual Frequency Radio Science experiment onboard Chandrayaan-2: a radio occultation technique to study temporal and spatial variations in the surface-bound ionosphere of the Moon
}

\author{
R. K. Choudhary ${ }^{1, *}$, K. R. Bindu ${ }^{2}$, Kumar Harshit ${ }^{2}$, Rahul Karkara ${ }^{2}$, \\ K. M. Ambili' ${ }^{1}$, T. K. Pant ${ }^{1}$, Devadas Shenoy ${ }^{2}$, Chandrakanta Kumar ${ }^{2}$, \\ N. Hemanth Kumar Reddy ${ }^{2}$, T. K. Rajendran ${ }^{3}$, M. Nazer ${ }^{1}$ and M. Shajahan ${ }^{1}$ \\ ${ }^{1}$ Space Physics Laboratory, Vikram Sarabhai Space Centre, Thiruvananthapuram 695022 , India \\ ${ }^{2}$ U.R. Rao Satellite Centre, Bengaluru 560 017, India \\ ${ }^{3}$ ISRO Telemetry, Tracking, and Command Network, Bengaluru 560 058, India
}

The Dual Frequency Radio Science experiment aboard Chandrayaan-2 uses the communication channel between orbiter and ground in radio occultation mode to study the temporal evolution of electron density in the lunar ionosphere. It consists of a highly stable $20 \mathrm{MHz}$ evacuated miniaturized crystal oscillator source, having a stability of the order of $10^{-11}$, which generates two coherent signals at the $X(8496 \mathrm{MHz})$ and $S(2240 \mathrm{MHz})$ bands of radio frequencies. The coherent radio signals, transmitted simultaneously from the satellite and received at the ground-based deep station network receivers would be used to study temporal and spatial variations in the lunar ionosphere. The major science objectives of the experiments include: (i) to study variations in the ionosphere/atmosphere of the Moon; (ii) to explore if the ionosphere of the Moon is omnipresent or has episodic appearances, and (iii) to confirm the source of ions in the lunar ionosphere - whether dusty or molecular.

Keywords: Chandrayaan, ionosphere, Moon, radio occultation.

THE Moon is considered to have a tenuous atmosphere; a relatively collision-less atmosphere, the entire envelope of which weighs not more than 100 tonnes $^{1}$. It has its origin derived from the external as well as internal processes $^{1-3}$. Mainly three types of external sources are considered as potential agents for the formation of the atmosphere at the Moon. These are solar radiations (photons), solar wind particles (plasma) and micro-meteors. When solar wind plasma (having energy of the order of

\footnotetext{
*For correspondence. (e-mail: rajkumar.choudhary@gmail.com)
}

$\mathrm{MeV}$ ) impinges on the lunar surface, it gets absorbed only to get neutralized, thermalized and re-emitted subsequently from the surface to build the atmospheric constituents. The presence of hydrogen $(\mathrm{H})$, helium $(\mathrm{He})$, neon $(\mathrm{Ne})$, and argon (Ar) atoms in the lunar exosphere can be attributed to this process ${ }^{1,4-8}$. Spectroscopic measurements have shown the existence of sodium $(\mathrm{Na})$, potassium $(\mathrm{K})$, and $\mathrm{H}_{2} \mathrm{O}$ as well in the lunar exosphere ${ }^{1,9,10}$. Additionally, when the Moon is in the Earth's magnetotail, which happens for about 4-5 days every lunation, the plasma sheet particles also contribute to the impacting plasma population thereby generating an atmosphere ${ }^{11}$. The internal sources of the lunar atmosphere include outgassing: the release of gases such as radon $(\mathrm{Rn})$ that originate from radioactive decay within the crust and mantle, constitute a source for the lunar atmosphere. The radioactive decay of $\mathrm{K}$ (40) into $\mathrm{Ar}$ (40), vented out from the lunar surface, is yet another source of the lunar atmosphere. Ground-based spectroscopic studies ${ }^{12}$ suggest evidence for atoms like $\mathrm{Si}, \mathrm{Ti}, \mathrm{Al}, \mathrm{Fe}, \mathrm{Mg}, \mathrm{Ca}$ and $\mathrm{O}$, which are considered to be the outcome of out-gassing from the lunar surface material; but the same have not yet been observed directly. In addition, gases like $\mathrm{CH}_{4}, \mathrm{NH}_{3}$ and $\mathrm{CO}_{2}$ are also likely to be present in small amounts ${ }^{9,10,13}$. The atmospheric loss processes include photo-ionization (once the gases are ionized they are picked up by solar wind and swept away), surface adsorption (re-implanted into the regolith as a result of the Moon's gravity), thermal escape and finally lost to space by solar radiation pressure.

The lunar atmosphere is the source of the ionosphere at the Moon. In the daytime lunar hemisphere, each atmospheric species is ionized by solar ultraviolet (UV) radiation. The plasma density at any point of time depends on the neutral density, its photo-ionization 
Table 1. Planetary missions with radio occultation (RO) experiment onboard. The stability is for the radio signals used in the experiment

\begin{tabular}{|c|c|c|c|c|}
\hline Spacecraft & Planetary body & Single/dual frequency & Frequency & Stability \\
\hline Mariner 4 & Mars & Single & $\mathrm{S} 2.3 \mathrm{GHz}$ & $\sim 10^{-11}$ \\
\hline Mariner 5 & Venus & $\begin{array}{l}\text { Dual Beacon } \\
\text { Single }\end{array}$ & $\begin{array}{c}49.8 \text { and } 423.3 \mathrm{MHz} \\
\text { S } 2.3 \mathrm{GHz}\end{array}$ & $\sim 10^{-10}$ \\
\hline Mariner 6 & Mars & Single & $\mathrm{S} 2.3 \mathrm{GHz}$ & $\sim 10^{-11}$ \\
\hline Mariner 7 & Mars & Single & $\mathrm{S} 2.3 \mathrm{GHz}$ & $\sim 10^{-11}$ \\
\hline Mariner 9 & Mars & Single & S $2.3 \mathrm{GHz}$ & $\sim 10^{-11}$ \\
\hline Mariner 10 & Mercury & Dual & $\begin{array}{l}\text { S } 2.3 \mathrm{GHz} \\
\text { X } 8.4 \mathrm{GHz}\end{array}$ & $\sim 10^{-11}$ \\
\hline Voyager 1 & Saturn & $\begin{array}{l}\text { Dual } \\
\text { Jupiter }\end{array}$ & $\begin{array}{l}\text { S } 2.3 \mathrm{GHz} \\
\text { X } 8.4 \mathrm{GHz}\end{array}$ & $\sim 10^{-12}$ \\
\hline MGS & Mars & Single & $\mathrm{X} 8.4 \mathrm{GHz}$ & $\sim 10^{-13}$ \\
\hline Mars Express & Mars & Dual & $\begin{array}{l}\text { S } 2.3 \mathrm{GHz} \\
\text { X } 8.4 \mathrm{GHz}\end{array}$ & $\sim 10^{-14}$ \\
\hline Venus Express & Venus & Dual & $\begin{array}{l}\text { S } 2.3 \mathrm{GHz} \\
\text { X } 8.4 \mathrm{GHz}\end{array}$ & $\sim 10^{-13}$ \\
\hline Akatsuki & Venus & Single & X $8.4 \mathrm{GHz}$ & $\sim 10^{-12}$ \\
\hline Galileo & Jupiter, Io & Single & $\mathrm{S} 2.295 \mathrm{GHz}$ & $\sim 10^{-12}$ \\
\hline Pioneer 10 & Jupiter & Single & $\mathrm{S} 2.3 \mathrm{GHz}$ & $\sim 10^{-10}$ \\
\hline Pioneer 11 & Jupiter & Single & S $2.3 \mathrm{GHz}$ & $\sim 10^{-10}$ \\
\hline Cassini & Saturn & Three & $\begin{array}{c}\mathrm{Ka} 32.0 \mathrm{GHz} \\
\text { X } 8.4 \mathrm{GHz} \\
\mathrm{S} 2.3 \mathrm{GHz}\end{array}$ & $\sim 10^{-13}$ \\
\hline Voyager 2 & Uranus & Dual & $\begin{array}{l}\text { S } 2.3 \mathrm{GHz} \\
\text { X } 8.4 \mathrm{GHz}\end{array}$ & $\sim 10^{-12}$ \\
\hline SELENE & Moon & Dual & $\begin{array}{l}\text { S } 2.3 \mathrm{GHz} \\
\text { X } 8.4 \mathrm{GHz}\end{array}$ & $\sim 10^{-11}$ \\
\hline New Horizon & Pluto & Dual & $\begin{array}{l}\text { S } 2.3 \mathrm{GHz} \\
\text { X } 8.4 \mathrm{GHz}\end{array}$ & $\sim 10^{-13}$ \\
\hline
\end{tabular}

cross-section and UV flux. Charge exchange reactions with the solar wind protons, solar wind electrons and photo-electron impact ionization are some of the other sources for the plasma in the lunar exosphere ${ }^{14}$. Charged dust particles near the lunar surface, being accelerated upwards to reach higher altitudes by the near-surface electric field can also cause an elevated plasma layer in the lunar ionosphere ${ }^{15}$. Solar wind sweeping across the interplanetary space is considered to be a major loss process of the plasma in the lunar exosphere ${ }^{5,14,16,17}$.

Much of the information about planetary/lunar ionosphere has been derived using the radio occultation (RO) technique, also known as the radio science technique. The first occultation experiment for the Moon was performed by the Pioneer-7 probe in 1966 (ref. 18). This experiment proved the existence of a thin ionosphere around the Moon. The electron density determined at that time was of the order of $10^{2}$ electrons $/ \mathrm{cm}^{3}$. The later Soviet missions, viz. Luna-19 and Luna-22 revealed the presence of a $10 \mathrm{~km}$ plasma layer having electron densities of the order of $0.5-2.0 \times 10^{3}$ electrons $/ \mathrm{cm}^{3}$ (ref. 19). The estimated columnar density of the lunar ionosphere falls in the range $3-5 \times 10^{14}$ electrons $/ \mathrm{cm}^{2}$. RO by SMART- 1 has also shown the existence of the Moon's ionosphere with columnar density of the order of $10^{13}$ electrons $/ \mathrm{cm}^{2}$ (ref. 20), which was further confirmed by the Selene ${ }^{21}$ and Chandrayaan-1 (ref. 14) measurements. This number, however, is highly variable as there are many factors which control electron density in the lunar ionosphere. Apart from solar conditions, another factor, that greatly influence lunar plasma production and distribution is the Earth's geomagnetic field. At times, when the Moon gets completely covered in the Earth's magneto-tail, it gets shielded from the direct impact of solar winds. During this period, the production of ions drastically reduces and so does the loss of plasma by solar-wind sweeping. The distribution of the plasma, in this case, is controlled by the Earth's geomagnetic field.

Rather surprisingly, even $\sim 50$ years after the Pioneer- 7 probe, we still lack sound knowledge of the lunar ionosphere and a complete picture on the tenuous lunar plasma is far from clear. In this context, technologically much superior missions like Chandrayaan-2 to the Moon could prove to be effective tools to study the concentration of plasma and/or neutrals as well as to generate a long-term map of electron distribution of the lunar ionosphere. This article describes the Dual Frequency Radio Science (DFRS) payload onboard Chandrayaan-2, which is a radio science experiment to study temporal and spatial evolution of plasma density in the lunar ionosphere. 


\section{Radio occultation technique}

The use of RO technique for studying ionosphere and atmosphere began with Mariner-4 occultation experiment on $\mathrm{Mars}^{22}$. The experiment conclusively proved that the surface pressure of Mars was about $4 \mathrm{hPa}$, instead of $100 \mathrm{hPa}$ as it was earlier believed to be (see Fjeldbo and Eshelman $^{23}$ and references therein). This startling finding threw a new dimension to our understanding about the Martian atmosphere. It was earlier believed that Mars had an $\mathrm{O}_{2}$-dominant atmosphere, owing to its surface pressure being of the order of $100 \mathrm{hPa}$. The experimental finding of $4 \mathrm{hPa}$ as surface pressure, combined with mass spectrometer measurements, concluded that Mars had a dominant $\mathrm{CO}_{2}$ atmosphere with almost no $\mathrm{O}_{2}$. This result paved the way for the RO experiment to become an integral part of all the subsequent planetary missions. Table 1 provides details of various planetary missions. Extending further, this technique is now used to study the Earth's ionosphere and atmosphere ${ }^{24-26}$.

The RO experiment relies on the principle that the phase of a radio signal, propagating from a satellite to the ground station, gets perturbed when it crosses through the planetary/lunar atmosphere or ionosphere. When the ground-based receivers receive radio signals from the satellite, the Earth's ionosphere constitutes a major source of the fluctuations in phase for the probing signals. As the trajectory of the spacecraft takes it behind the planet as seen from Earth, there is occultation by the planet's atmosphere/ionosphere and an additional phase shift is introduced in the probing signals. Figure 1 shows a schematic of the RO experiment. Under the assumption that during the period while the spacecraft occults with the planetary ionosphere, there is no significant change in phase fluctuation due the Earth's ionosphere, the extra phase shift in the probing radio signals can be safely attributed to planetary atmosphere/ionosphere only. The extra phase changes can be computed with reasonable

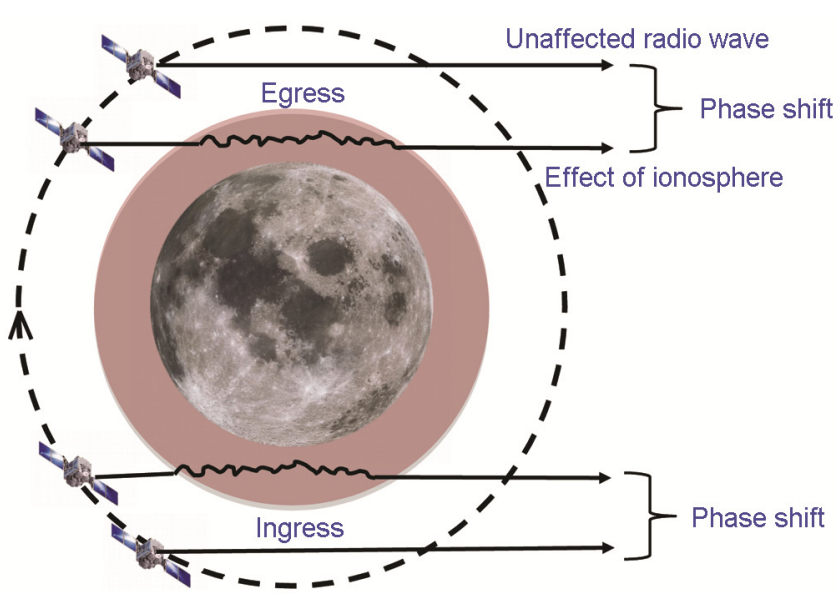

Figure 1. Schematic diagram for the radio occultation technique. accuracy using high-sensitive ground-based receivers. In standard practice, radio signals in the $\mathrm{S}(\sim 2.5 \mathrm{GHz})$ and $\mathrm{X}$ $(\sim 8.4 \mathrm{GHz})$ bands, which are normally used for telemetry, tracking and command (TTC) purposes, are used for radio probing as well. To use the dual frequencies for RO studies, however, proper phase coherence between the two frequencies must be maintained. It is worth mentioning that using RO technique, the vertical profile of the atmosphere as well as the ionosphere can be derived with a height resolution as high as $100 \mathrm{~m}$, which no other passive sounding instruments can achieve ${ }^{27}$.

\section{Dual frequency sounding: advantages and uniqueness}

The RO technique involves estimation of additional phase changes in the satellite's radio signal due to the planetary medium. The signals which get occulted by the planetary medium, however, have to cross through the Earth's environment as well while being received at the ground via the deep station network antennas. There would be an extra phase shift in the radio signal due to the Earth's medium, which introduces some extra time delay in the signal. The phase change in radio signals due to the intervening Earth's environment, however, could be far more than the phase change due to the planetary medium. An accurate estimation of parameters in the planetary medium, therefore, depends on the accurate estimation of extra phase changes in the radio signals due to the medium other than the planetary medium.

Total phase change in radio signals recorded at the ground includes Doppler due to relative motion between the occulting planet, Earth and satellites (called theoretical Doppler), Doppler due to bending of radio signals due to the Earth's neutral atmosphere and dispersion in radio signals due to the Earth's ionosphere, in addition to phase change due to the occulting planet's atmosphere and ionosphere. It can be represented as

$$
\phi_{t}=\phi_{\mathrm{th}}+\phi_{\mathrm{ea}}+\phi_{\mathrm{ei}}+\phi_{\mathrm{pa}}+\phi_{p i}
$$

where $\phi_{t}$ is the total phase change in radio signals received at the ground. $\phi_{\mathrm{th}}$ the phase change due to relative motion between Earth-planet-satellite. $\phi_{\text {ea }}$ the phase change due to the Earth's atmosphere, $\phi_{\mathrm{ei}}$ the phase change due the Earth's ionosphere, $\phi_{\mathrm{pa}}$ the phase change due to the planet's atmosphere and $\phi_{\mathrm{pi}}$ is the phase change due to the planet's ionosphere.

The separation of components of phase change in radio signals due to the occulting medium from the total phase change recorded at the ground is an important step in the analysis process, as it constitutes a major source of error in the magnitude of inverted electron density/neutral density profiles. It requires precise determination of the satellite's orbit (or $\phi_{\mathrm{th}}$ ), along with an accurate estimation 
of phase change due to the Earth's ionosphere and atmosphere $\left(\phi_{\mathrm{ea}}+\phi_{\mathrm{ei}}\right)$. Using various techniques like Delta$\mathrm{DOR}^{28}$ and precise ephemeris, the theoretical Doppler $\left(\phi_{\mathrm{th}}\right)$ can be estimated accurately to some fairly good extent ${ }^{29}$.

Once the phase change due to relative motion between the Earth-planet-satellite system is established, the task is to separate Doppler due to the Earth's atmospheric and ionospheric components. The phase change in radio signals due to atmosphere/ionosphere depends on the refractive index of the medium, which is given in terms of refractivity as ${ }^{30}$

$N=(n-1) \times 10^{6}=77.6 \frac{P}{T}+3.73 \times 10^{5} \frac{P_{w}}{T^{2}}-4.03 \times 10^{7} \frac{n_{\mathrm{e}}}{f^{2}}$,

where $N$ is the refractivity of the medium, $n$ the refractive index, $P$ and $T$ are pressure and temperature of the dry atmosphere respectively and $P_{\mathrm{w}}$ is the partial pressure of water vapour. $n_{e}$ and $f$ represent electron density and frequency of the probing radio signals respectively. The first two terms on the right side of the above equation account for refractivity of a medium due to neutral atmosphere. The bending of radio signals due to the neutral medium, therefore, does not depend upon the frequency of the signals. It depends only on the neutral density. The third term, which is inversely proportional to the square of frequency, is due to the ionosphere which is a dispersive medium. The radio signals passing through this medium would have varying degree of dispersion due to varying frequencies. A radio wave of lower frequency therefore would suffer higher dispersion and vice-versa. Excluding oscillator drifts and instabilities, the frequency shift for a one-way (spacecraft-to-Earth) radio link due to plasma is given as ${ }^{30}$

$$
\Delta f=f-f_{0}=-\frac{f_{0}}{c} \frac{\mathrm{d} r}{\mathrm{~d} t}+\frac{40.31}{c} \frac{1}{f_{0}} \frac{\mathrm{d}}{\mathrm{d} t} \int_{s / c}^{\text {Earth }} n_{\mathrm{e}} \mathrm{d} r,
$$

where $f_{0}$ is the carrier frequency, $f$ the satellite frequency recorded at the receiver. $\mathrm{d} r / \mathrm{d} t$ represents the rate of change of distance between transmitter and receiver, and $c$ is the speed of light. The integral of the electron density $n_{e}(r)$ along the propagation path $\mathrm{d} r$ of the radio wave from the spacecraft to the Earth is the total electron content (TEC) of the medium.

In the above equation, the first term on the right-hand side is the classical Doppler shift and the second term is the dispersive propagation effect of radio waves in the ionized media, which is inversely proportional to $f_{0}$. Obviously, a larger Doppler shift is measured for the $\mathrm{X}$-band of the radio frequency than the $\mathrm{S}$ band. The lower S-band frequency, however, is more sensitive to dispersive frequency shifts.
It is not possible to separate classical and dispersive frequency shifts from the observed total change in frequency $\Delta f$ at either $f_{s}$ or $f_{x}$ (the S-band or X-band carrier frequency respectively) alone. For the two phase-coherent downlinks at the X- and S-bands with a constant transponder ratio of $f_{x}=f_{s}$, the differential Doppler can be estimated by

$$
\Delta f_{s}-\frac{f_{s}}{f_{x}} \Delta f_{x}
$$

where $\Delta f_{s}$ and $\Delta f_{x}$ are the observed Doppler shifts at the $\mathrm{S}$ and $\mathrm{X}$ band respectively. Now it is possible to isolate the dispersive frequency shift using relations

$$
\Delta f_{s}-\frac{f_{s}}{f_{x}} \Delta f_{x}=\frac{40.31}{c} f_{s}\left[\frac{1}{f_{s}^{2}}-\frac{1}{f_{x}^{2}}\right] \frac{\mathrm{d}}{\mathrm{d} t} \int_{\mathrm{s} / \mathrm{c}}^{\text {Earth }} n_{\mathrm{e}} \mathrm{d} r .
$$

The differential Doppler effect calculated is then used to determine the changes in electron content. All oscillator drifts and relative velocities get eliminated in this process.

For the RO experiment, two radio signals which are widely separated in their frequencies generated using a highly stable oscillator are ideal. However, if two separate coherent radio frequencies are not available, even a single frequency (in downlink mode) has been used to derive electron density/neutral density in the ionospheric/ atmospheric region. It is worth mentioning that one of the most successful RO experiments was done using Mars Global Surveyor (MGS), which had only one frequency at the $\mathrm{X}$ band $^{31}$. MGS gave approximately 5600 profiles of electron density on Mars. It is to be noted that the total number of electron density profiles available on Mars, till date, is about 6500 only. Even in the case of Chandrayaan-1, where TTC signals at the X-and S-bands were non-coherent, the single $\mathrm{S}$-band radio frequency was successfully used to study the origin of atmosphere and ionosphere of the Moon ${ }^{14}$. Table 1 provides frequencies and mode of operation used in various planetary missions. It is, however, worth mentioning that with the single-frequency measurements, electron density less than $500 \mathrm{~cm}^{-3}$ cannot be estimated unambiguously ${ }^{31}$.

\section{Scientific objectives of the DFRS experiment}

\section{Scientific background}

Initial evidences for plasma in the lunar exosphere came from passive stellar radio experiments. Large angular shifts in radio waves were observed when stellar radio signals were occulted by the dayside lunar limb $b^{32,33}$. Such shifts in the radio signals were ascribed to the existence of the lunar ionosphere with electron density of the order 
of $1000 \mathrm{~cm}^{-3}$ and thickness of several kilometres. Confirmation for the existence of a thin ionosphere around the Moon later came from the active radio soundings. The RO experiment by Pioneer-VII estimated electron density of the order of $4 \times 10^{7}$ electron $/ \mathrm{m}^{3}$ in the lunar ionosphere ${ }^{18}$. Evidence for the presence of the lunar ionosphere came from in situ measurements like charged particle lunar environment experiment (CPLEE) onboard the US Apollo-14 mission too. The plasma density estimated by CPLEE was $\sim 10^{4}$ electrons $/ \mathrm{cm}^{3}$, covering several hundred metres of altitude during the lunar daytime ${ }^{34}$. Later measurements using DFRS experiments by the Soviet Luna-19 and Luna-22 missions further confirmed the presence of a $30 \mathrm{~km}$ plasma layer characterized by an electron number density of $0.5-2 \times 10^{3}$ electrons $/ \mathrm{cm}^{3}$ (ref. 19). Large variations in the magnitude of peak plasma density were reported. At 14:05 local time (LT), a peak plasma density as high as $\sim 2000 \mathrm{~cm}^{3}$ was shown to exist in the lunar ionosphere, while the same then became $400 \mathrm{~cm}^{-3}$ by 19:04 LT (ref. 19). In addition, solar activity and position of the Moon with respect to the Earth's magento-tail were the other factors which were shown to have control on plasma density in the lunar ionosphere.

Most of the profiles from the Luna-22 missions were for the near-equatorial low-latitude regions of the lunar ionosphere. Recently, the SELENE (SELenological and ENgineering Explorer) spacecraft of Japan conducted a series of RO measurements to determine the line-of-sight electron column concentration, or TEC, above the limb of the Moon as a function of tangent height ${ }^{21,35}$, particularly in the polar regions of the Moon. These measurements also confirmed the presence of a lunar ionosphere above the sunlit lunar surface, with peak electron density of $300 \mathrm{~cm}^{-3}$ occurring around an altitude of $15 \mathrm{~km}$ above the surface.

The S-band single-frequency RO measurements by Chandrayaan-1 further confirmed the presence of plasma in the lunar ionosphere below $40 \mathrm{~km}$ altitude with a peak density of the order of $\sim 300 \mathrm{~cm}^{-3}$ (ref. 14). These values are in broad agreement with those inferred from earlier RO measurements, where the electron densities observed were of the order of 500-1000 $\mathrm{cm}^{-3}$ (refs 19, 32 and 36). However, the process of generation of lunar ionosphere through photo-ionization of neutral gas has been found to be inadequate to account for the observed ionization densities. It was argued that though the photo-ionization of ambient inert gases (Ar, He and $\mathrm{Ne}$ ) could lead to an appreciable concentration of plasma at the lunar surface, the pick-up of ions by solar winds results in their complete sweeping leading to the near-negligible presence of plas$\mathrm{ma}$ in the lunar ionosphere ${ }^{17}$. Not more than a few electrons per cubic centimetre shown to exist in the lunar ionosphere, if photo-ionization was considered a potential source. As an alternative, a concept of dusty plasma was put forward by Stubbs ${ }^{15}$. The electrical charging of exosphere by levitating dust leading to increased ionization was proposed as a possible mechanism for the observed increased ionization. However, recent measurements of dust from LAMP (Lyman Alpha Mapping Project) aboard Lunar Reconnaissance Orbiter (LRO) have shown that the surface concentration of dust in the Moon could be not more than $10^{-5}$ particles $\mathrm{cm}^{-3}$ (ref. 37), while Stubbs ${ }^{15}$ assumed dust surface density of about $10^{-1}$ particles $\mathrm{cm}^{-3}$, about four orders of magnitude higher than the LAMPRO measurements. The origin of plasma source on the Moon was revisited using a photochemical model, which included photo-production and loss reactions of 16 ions, charge exchange reactions with solar wind protons, solar wind electrons, and photo-electron impact ionization ${ }^{14}$ and it was shown that the lunar ionosphere can have ions of molecular origins $\left(\mathrm{H}_{2} \mathrm{O}^{+}, \mathrm{CO}_{2}^{+}\right.$and $\left.\mathrm{H}_{3} \mathrm{O}^{+}\right)$, rather than inert ions $\left(\mathrm{Ar}^{+}, \mathrm{Ne}^{+}, \mathrm{He}^{+}\right)$.

\section{Objectives}

Since the Apollo era, there have been several studies of the lunar ionosphere starting ${ }^{14,18}$. All these have given us an understanding that the lunar ionosphere consists of a surface-bound plasma layer, extending up to $40 \mathrm{~km}$ in altitude, with peak plasma density varying between $\sim 2000$ $\sim 300 \mathrm{~cm}^{-3}$ depending upon the solar zenith angle and solar activity conditions. A well-established/understood physical mechanism satisfactorily explaining all the observations, including the high electron concentrations in the lunar environment, however, is still elusive. There have been competing theories for the possible existence of plasma in the lunar exosphere, which is believed to have a thin presence of neutral atmosphere. One such theory deals with photo-emission of electrons from suspended dust particles ${ }^{15}$. Upward acceleration of charged lunar dust clouds, accompanied by substantial number of electrons caused by the near-surface electric field, could be a possible source for enhanced ionization. The dustelectron hypothesis, however, is yet to be established experimentally. Theoretically, the reversal in the lunar surface potential over higher lunar latitudes should lead to efficient removal of electrons by the solar wind towards the nightside of the Moon. However, there have been no experimental evidences either proving or disproving it.

A recent study has shown that there exists a possibility that the Moon could have plasma of molecular origin ${ }^{14}$. Using a photo-chemical model, which considered production of electrons due to solar wind apart from extreme ultra violet (EUV) radiations, the study reported that in the absence of loss of plasma due to solar wind sweeping, the peak plasma density at the lunar ionosphere could be as high as $10^{5} \mathrm{~cm}^{-3}$ (ref. 14). It is the solar wind sweeping which renders the Moon bereft of plasma. In this respect, it is worth mentioning that during the period of lunation, when the Moon remains under the Earth's magneto-tail, 
loss of plasma due to solar-wind sweeping does not take place. In that case, electron density in the lunar ionosphere could be far more than $300 \mathrm{~cm}^{-3}$, a typical plasma density which the Moon is believed to have. Therefore, a systematic analysis is needed to study the temporal evolution of plasma in the lunar ionosphere. In addition, the impact of solar activity variations on the lunar ionosphere is still undocumented. In this context, the DFRS payload on Chandrayaan-2 will answer several questions, important among which are the following: (i) To study temporal variation of the lunar ionosphere in the vicinity of the lunar surface under varying solar conditions. (ii) To portray the lunar ionosphere, especially its morphology and the TEC. (iii) To understand the genesis of plasma in the lunar environment and examine the role of molecular and/or dusty plasma in the overall ion budget. (iv) To examine the effect of the Earth's magnetosphere and magneto-tail on the production and distribution of plasma in the lunar ionosphere.

\section{DFRS: system specifications and technical details}

\section{Technical specifications}

Table 2 lists the technical specifications of the DFRS payload. It uses evacuated miniaturized crystal oscillator (EMXO) as a master oscillator to generate radio signals at $20 \mathrm{MHz}$, and further divided to generate coherent dual frequencies at the $\mathrm{X}$ - and S-bands.

\section{Configuration}

The DFRS experiment requires coherent generation of S- and X-band highly stable carrier. To achieve the same, it is required to use a highly stable reference oscillator source for carrier generation. The EMXO was selected for this purpose, which provides short-term stability (Allen deviation) of better than $10^{-11}$ in $0.1 \mathrm{sec}$ (Figure 2)

Table 2. Technical specifications of DFRS payload on Chandrayaan-2

\begin{tabular}{ll}
\hline Frequency & $\begin{array}{l}\text { Coherent X-band and S-band } \\
(8496 \text { and 2240 MHz) }\end{array}$ \\
Reference frequency & $\begin{array}{l}20 \mathrm{MHz} \\
\text { Evacuated miniaturized crystal } \\
\text { oscillator }(\text { EMXO) }\end{array}$ \\
& Better than $1 \mathrm{e}^{-11}(1 \mathrm{sec})$ \\
Reference short-term stability & \\
$\begin{array}{l}\text { Transmitted EIRP } \\
\text { S-band }\end{array}$ & $\begin{array}{l}4 \mathrm{dBW} \\
\text { X-band }\end{array}$ \\
$\begin{array}{l}\text { Received CNDR } \\
\text { S-band } \\
\text { X-band }\end{array}$ & $49.4 \mathrm{dBW}-\mathrm{Hz}$ \\
Total power consumption & $55 \mathrm{~dB}-\mathrm{Hz}$ \\
\hline
\end{tabular}

with very low power ( $0.5 \mathrm{~W}$ after stabilization) and low mass.

To generate coherent signals, the output of $20 \mathrm{MHz}$ basic EMXO source is fed to a two-way power divider. The two outputs are further fed to the carrier-generating circuits using phase lock loop (PLL) synthesizers, one giving the output at S-band and the other at X-band. Figure 3 shows block diagram of the DFRS payload.

Both the carrier-generating circuits are similar in function. The carrier is generated directly at the required band using an X-band/S-band voltage controlled oscillator (VCO), and by locking it to the reference EMXO using PLL technique. The carrier at the required frequency is generated by applying appropriate control voltage to the VCO from the PLL loop. The VCO output is passed through a directional coupler whose coupled port output

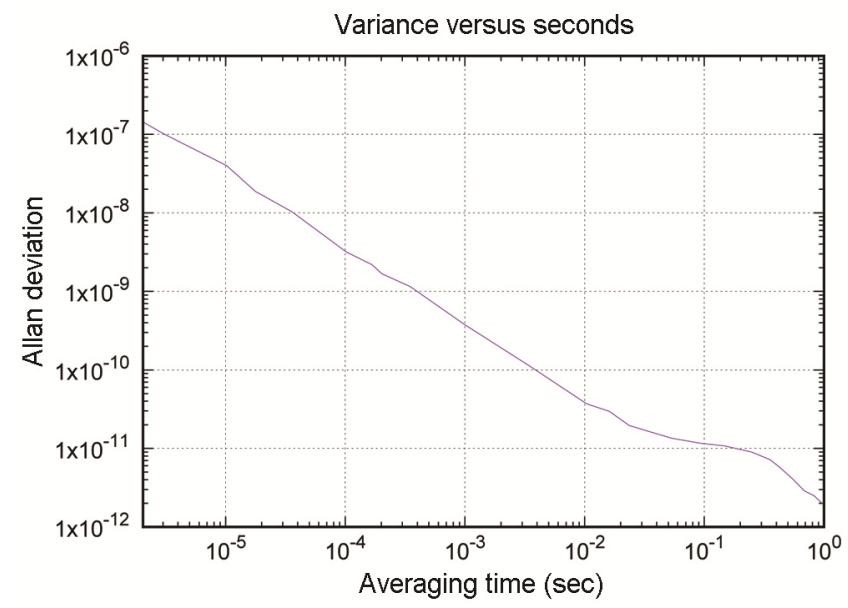

Figure 2. Allan Variance of the EMXO receiver after $20 \mathrm{ON} / \mathrm{OFF}$ cycles (each cycle of $15 \mathrm{~min} \mathrm{ON}$ and $45 \mathrm{~min} \mathrm{OFF}$ ).

Table 3. Link estimates for the S- and X-bands of radio signals for DFRS payload on Chandrayaan-2

$\begin{array}{ll}\text { S-band } & \\ \text { Amplifier power O/P } & -10 \mathrm{dBW} \\ \text { Onboard loss } & 2 \mathrm{~dB} \\ \text { Onboard antenna gain } & 2 \mathrm{dBi} \\ \text { Transmit EIRP } & -10 \mathrm{dBW} \\ \text { Path loss } & 211.6 \mathrm{~dB} \\ \text { Ground station G/T } & 36 \mathrm{~dB} /{ }^{\circ} \mathrm{K}(32 \mathrm{~m} \mathrm{IDSN}) \\ \text { Antenna pointing loss } & 0.2 \mathrm{~dB} \\ \text { Received CNDR } & 42.8 \mathrm{~dB}-\mathrm{Hz} \\ & \\ \text { X-band } & -14 \mathrm{dBW} \\ \text { Amplifier power O/P } & -24 \mathrm{dBW} \\ \text { O/P of 10 dB coupler } & 2 \mathrm{~dB} \\ \text { Onboard loss } & 33 \mathrm{dBi} \\ \text { Onboard antenna gain } & 7 \mathrm{dBW} \\ \text { Transmit EIRP } & 223.2 \mathrm{~dB} \\ \text { Path loss } & 0.4 \mathrm{~dB} \\ \text { Antenna pointing loss } & 42 \mathrm{~dB} /{ }^{\circ} \mathrm{K}(32 \mathrm{~m} \text { IDSN) } \\ \text { Ground station G/T } & 54 \mathrm{~dB}-\mathrm{Hz} \\ \text { Received CNDR } & \end{array}$




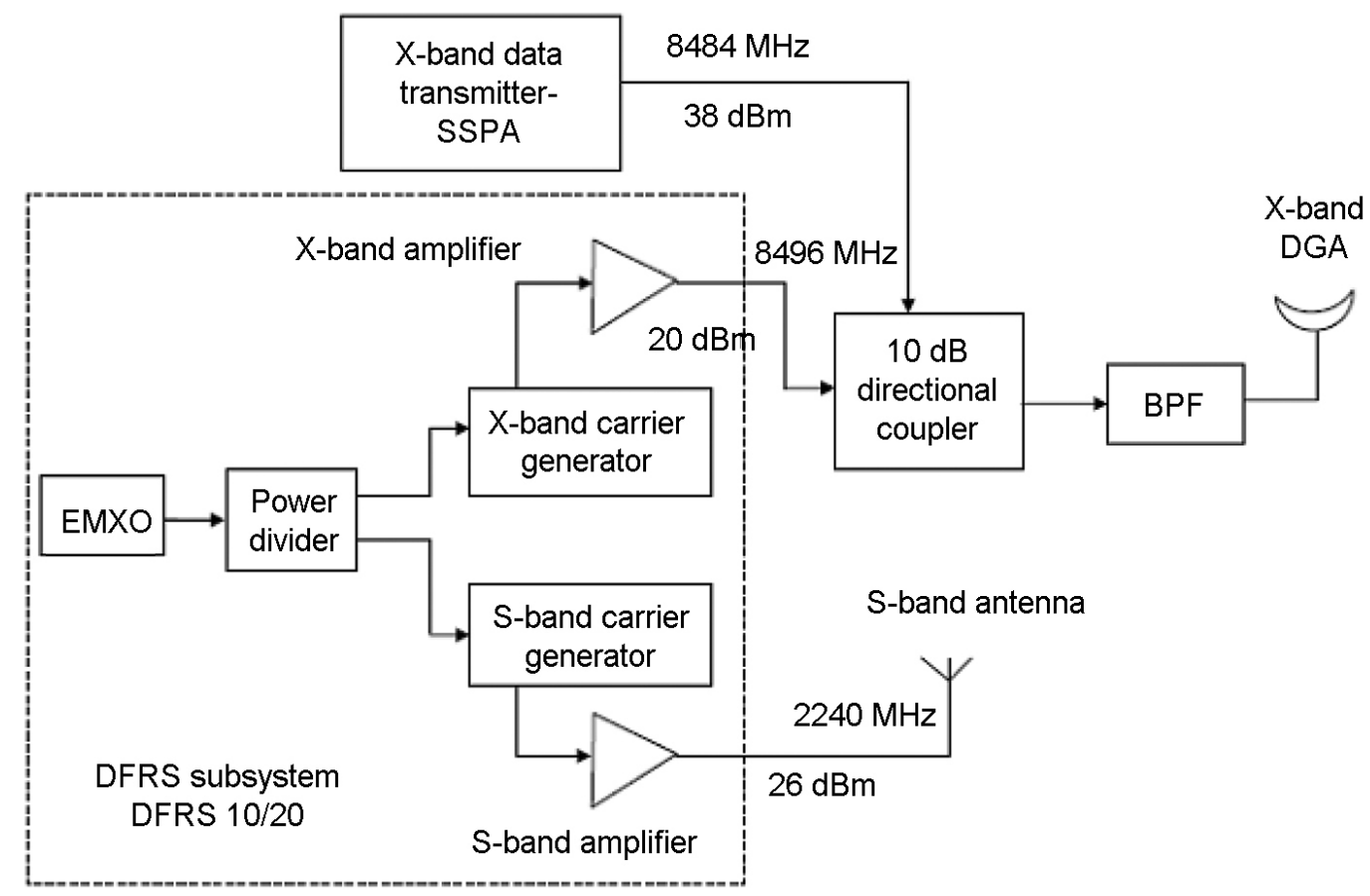

Figure 3. Schematic diagram showing different components in the DFRS payload of the orbiter.

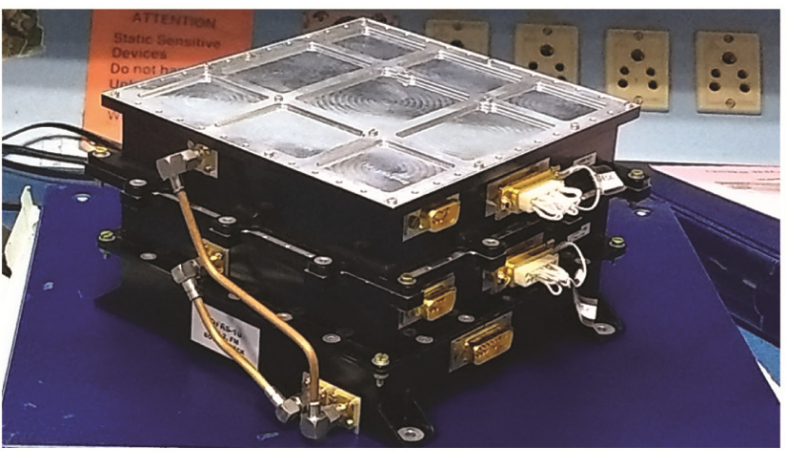

DFRS -10

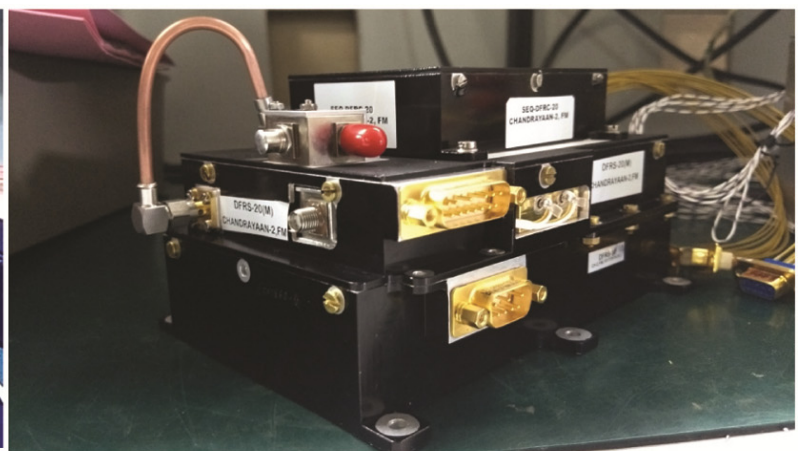

DFRS -20

Figure 4. Flight model version of DFRS-10 and DFRS-20 on Chandrayaan-2.

is given to the RF input port of the PLL synthesizer PE97042, the main port output being used as the carrier. A lock status is provided from the PLL synthesizer, which is processed to act as the lock indication telemetry.

The S-band carrier of frequency $2240 \mathrm{MHz}$ is amplified to a level of $26 \mathrm{dBm}$ and is transmitted through a separate S-band patch antenna having a gain of about $2 \mathrm{dBi}$. The X-band signal is also amplified to a power level of $20 \mathrm{dBm}$, and then applied to the coupled port of a $10 \mathrm{~dB}$ directional coupler. The main port of the directional coupler is fed from the X-band data transmitter. The frequency of X-band signal for the DFRS experiment is selected to be $8496 \mathrm{MHz}$. The combined signal from the directional coupler is transmitted through the onboard $0.7 \mathrm{~m}$ reflector antenna.
Hardware is realized as two packages, viz. DFRS 10 which houses the X-and S-band carrier generators, and DFRS 20 which houses the X-band and S-band amplifiers. Figure 4 shows the flight models of DFRS 10 and DFRS 20.

\section{Link estimates and measurement accuracy}

Table 3 gives the link estimates for S- and X-bands of radio signals. The carrier to noise density ratio (CNDR) for the S- and X-bands is 42.8 and $54 \mathrm{~dB}-\mathrm{Hz}$ respectively. As the accuracy in the measurements of columnar electron density is dependant on the accuracy of differential phase measurements, CNDR of the radio signals plays an 
important role as it controls the accuracy of phase measurements. Considering TEC of the lunar ionosphere to be of the order of $6.0 \times 10^{13} \mathrm{~m}^{-2}$, which is about $20 \%$ of the total TEC on the Moon ${ }^{14,21}$, the required CNDR for the $\mathrm{S}$ - and $\mathrm{X}$-bands of radio signals was estimated to be 34 and $22 \mathrm{~dB}-\mathrm{Hz}$ respectively ${ }^{38}$. These values are well within the link estimates of the DFRS payload.

Another factor which may affect the accuracy of differential phase measurements is the Earth's ionosphere. A study using dual-frequency GPS satellite measurements over Japan $^{39}$, has shown that the root-mean-square fluctuations in the Earth's TEC could be $\sim 10^{14} \mathrm{~m}^{2}$, which is of the same order as that of the lunar TEC. However, since the GPS measurements were carried out at a coarse time resolution of $30 \mathrm{sec}$, and also since a normal dualfrequency GPS receiver system uses OCXO-based crystal oscillator, which has stability of the order of $10^{-6}$ only, the phase fluctuations in radio signals due to the Earth's ionosphere cannot be estimated correctly based on such a study. Estimation of the Earth's TEC fluctuations using a receiver system having better stability is necessary to correctly estimate terrestrial contributions for the Moon's ionospheric measurements.

\section{Summary}

The DFRS experiment aboard Chandrayaan-2 aims to study variations in the ionosphere/atmosphere of the Moon. It also aims to explore if the Moon's ionosphere is omnipresent or has episodic appearances, and to confirm if the plasma is of molecular origin or dusty. It uses the communication channel between orbiter and ground in RO mode for this purpose. The DFRS consists of a highly stable $20 \mathrm{MHz}$ EMXO source, having stability of the order of $10^{-11}$, which generates two coherent signals at the $\mathrm{X}(8496 \mathrm{MHz})$ and $\mathrm{S}(2240 \mathrm{MHz})$ bands of radio frequencies. The coherent radio signals, transmitted simultaneously from the satellite, and received at the ground-based deep space network receivers would be used to study temporal and spatial variations in the lunar ionosphere.

1. Stern, S. A., The lunar atmosphere: history, status, current problems, and context. Rev. Geophys., 1999, 37, 453-492.

2. Killen, R. M. and Ip, W., The surface-bound atmospheres of Mercury and the Moon. Rev. Geophys., 1999, 37, 361-406.

3. Mendillo, M., Baumgardner, J. and Wilson, J., Observational test for the solar wind of the Moon's sodium atmosphere. Icarus, 1999, 137, 23-25.

4. Hinton, F. L. and Taeusch, D., Variation of the lunar atmosphere with the strength of the solar wind. J. Geophys. Res., 1964, 69(7), 1341-1347.

5. Johnson, F. S., Lunar atmosphere. Rev. Geophys. Space Phys., 1971, 9, 813-823.

6. Hodges, R. R., Hoffman, J. H., Johnson, F. S. and Evans, D. E., Lunar atmosphere composition results from Apollo 17. Proc. Lunar Sci. Conf., 1973, 2875, 2865-2875.
7. Hodges, R. R., Methods for Monte Carlo simulation of the exospheres of the Moon and Mercury. J. Geophys. Res., 1980, 85, 164170 .

8. Daily, W. D., Barker, W. A., Clark, M., Dyal, P. and Parkin, C. W., Ionosphere and atmosphere of the Moon in the geomagnetic tail. J. Geophys. Res., 1977, 82(33), 5441-5451.

9. Sridharan, R., Ahmed, S. M., Tirtha Pratim Das, Sreelatha, P., Pradeepkumar, P., Neha Naik and Supriya, G., 'Direct' evidence of water $\left(\mathrm{H}_{2} \mathrm{O}\right)$ in the sunlit lunar ambience from CHASE on MIP of Chandrayan I. Planet Space Sci., 2010, 58.

10. SridharanR., Ahmed, S. M., Das, T. P., Sreelatha, P., Pradeepkumar, P., Naik, N. and Supriyas, G., The sunlit lunar atmosphere: a comprehensive study by CHASE on the Moon impact probe of Chandrayan-I. Planet. Space Sci., 2010, 58, 1567-1577.

11. Wilson, J. K., Mendillo, M. and Spence, H. E., Magnetospheric influence on the Moon's exosphere. J. Geophys. Res., 2006, 111.

12. Flynn, B. C. and Stern, S., A spectroscopic survey of metallic species abundances in the lunar atmosphere. Icarus, 1996, 124, 530-536.

13. Hoffman, J. H., Lunar atmospheric composition experiment. Final Report, 1 June 1971-30 September 1975 Texas University at Dallas, USA, 1975.

14. Choudhary, R. K., Ambili, K. M., Choudhury, S., Dhanya, M. B. and Bhardwaj, A., On the origin of the ionosphere at the Moon using results from Chandrayaan-1 S-band radio occultation experiment and a photochemical model. Geophys. Res. Lett., 2016, 43.

15. Stubbs, T. J., Glenar, D. A., Farrell, W. M., Vondrak, R. R., Collier, M. R., Halekas, J. S. and Delory, G. T., On the role of dust in the lunar ionosphere. Planet Space Sci., 2011, 59, 1659-1664.

16. Hodges, R. R., Hoffman, J. H. and Johnson, F. S., The lunar atmosphere. Icarus, 1974, 21, 415-426.

17. Bauer, S. J., Limits to a lunar ionosphere. Anz. Abt. II, 1996, 133, 17-21.

18. Pomalaza-Díaz, J. C., Measurement of the Lunar Ionosphere by Occultation of the Pioneer VII Spacecraft. Department of Electrical Engineering, Stanford University, USA, 1967.

19. Vyshlov, A. S., Preliminary results of circumlunar plasma research by the Luna 22 spacecraft. In Space Research XVI (ed. Rycroft, M. J.), 1976, pp. 945-949.

20. Pluchino, S., Schilliro, F., Salerno, E., Pupillo, G., Maccaferri, G. and Cassaro, P., Radio occultation measurements of the lunar ionosphere. Mem. S.A. Lt. Suppl., 2008, 12(53).

21. Imamura, T. et al., Radio occultation measurement of the electron density near the lunar surface using a subsatellite on the SELENE mission. J. Geophys. Res., 2012, 117, A06303.

22. Kliore, A., Cain, D. L., Levy, G. S., Eshleman, V. R., Fjeldbo, G. and Drake, F. D., Occultation experiment: Results of the first direct measurement of Mars's atmosphere and ionosphere. Science, 1965, 149(3689), 1243-1248.

23. Fjeldbo, G. and Eshelman, V., The atmosphere of Mars analyzed by integral inversion of the Mariner IV occultation data. Planet. Space Sci., 1968, 16, 1035-1059.

24. Kursinski, E. R., Hajj, G. A., Schofield, J. T., Linfield, R. P. and Hardy, K. R., Observing Earth's atmosphere with radio occultation measurements using the Global Positioning System. J. Geophys. Res.: Atmos., 1997, 102(D19), 23429-23465.

25. Wickert, J. et al., Atmosphere sounding by GPS radio occultation: first results from CHAMP. Geophys. Res. Lett., 2001, 28(17), 3263-3266.

26. Schreiner, W., Rocken, C., Sokolovskiy, S., Syndergaard, S. and Hunt, D., Estimates of the precision of GPS radio occultations from the COSMIC/FORMOSAT-3 mission. Geophys. Res. Lett., 2007, 34.

27. Karayel, E. T. and Hinson, D., Sub-Fresnel scale vertical resolution in atmospheric profiles from radio occultation. Radio Sci., 1997, 32, 411-423.

28. Curkendall, D. W. and Border, J. S., Delta-DOR: the onenanoradian navigation measurement system of the deep space 
network - history, architecture, and componentry. In The Interplanetary. Network Progress Report, 2013, vol. 42, p. 193.

29. Krisher, T. P., Parametrized post-Newtonian gravitational redshift. Phys. Rev. D, 1993, 48(10), 4639.

30. Pätzold, M. et al., MaRS: Mars express orbiter radio science. In Mars Express: The Scientific Payload, 2004, vol. 1240, pp. 141163.

31. Hinson, D. P., Simpson, R. A., Twicken, J. D., Tyler, G. L. and Flasar, F. M., Initial results from radio occultation measurements with Mars Global Surveyor. J. Geophys. Res.: Planets, 1999, 104(E11), 26997-27012.

32. Elsmore, B., Radio observations of the lunar atmosphere. Philos. Mag., 1957, 2, 1040-1046.

33. Andrew, B. H., Branson, N. J. B. A. and Wills, D., Radio observations of the Crab nebula during a lunar occultation. Nature, 1964, 203, 171-173.

34. Reasoner, D. L. and Burke, W., Characteristic of the lunar photoelectron layer in the geomagnetic tail. J. Geophys. Res., 1972, 77, 6671-6687.

35. Ando, H. et al., Dual-spacecraft radio occultation measurement of the electron density near the lunar surface by the SELENE mission. J. Geophys. Res.: Space Phys., 2012, 117(A8).

36. Vyshlov, A. S. and Savich, N. A., Observations of radio source occultations by the moon and the nature of the plasma near the moon. Cosmic Res., 1979, 16, 551-556.
37. Feldman, P. D. et al., Upper limits for a lunar dust exosphere from far-ultraviolet spectroscopy by LRO/LAMP. Icarus, 2014, 233, 106-113.

38. Imamura, T. et al., The possibility of studying the lunar ionosphere with the SELENE radio science experiment. Earth, Planets Space, 2008, 60(4), 387-390.

39. Noguchi, K., Imamura, T., Oyama, K.-I. and Saito, A., Application of the GPS network to estimate the effect of the terrestrial ionosphere on the radio occultation measurements of planetary ionospheres. Radio Sci., 2001, 36(6), 1607-1613.

ACKNOWLEDGEMENTS. We thank the ADCOS committee of ISRO, India for down-selecting DFRS as a payload for the Chandrayaan-2 mission. We also thank members of several review committees, specially the technical review committee chaired by Shri K. Kiran Kumar, ex-Chairman, ISRO, and CDR committee chaired by Dr Anil Bhardwaj, Director, PRL, Ahmedabad for their constructive criticisms and valuable suggestions, and M. Chandrasekhar (Centum Rakon, Bengaluru) for Figure 2.

Received and accepted 27 August 2019

doi: $10.18520 / \mathrm{cs} / \mathrm{v} 118 / \mathrm{i} 2 / 210-218$ 